OPEN ACCESS

Edited by:

Daniela Puzzo,

University of Catania, Italy

Reviewed by:

Utpal Das,

University of California, San Diego,

United States

Mario Buffelli,

University of Verona, Italy

*Correspondence:

Pu Wang

wangpu@mail.neu.edu.cn

tThese authors have contributed equally to this work

Received: 02 October 2020 Accepted: 17 December 2020

Published: 15 January 2021

Citation:

Zhang S-Q, Cao L-L, Liang $Y-Y$ and Wang P (2021) The Molecular Mechanism of Chronic High-Dose Corticosterone-Induced Aggravation of Cognitive Impairment in APP/PS1 Transgenic Mice. Front. Mol. Neurosci. 13:613421. doi: 10.3389/fnmol.2020.613421

\section{The Molecular Mechanism of Chronic High-Dose Corticosterone-Induced Aggravation of Cognitive Impairment in APP/PS1 Transgenic Mice}

\author{
Shen-Qing Zhang ${ }^{\dagger}$, Long-Long Cao ${ }^{\dagger}$, Yun-Yue Liang and Pu Wang* \\ College of Life and Health Sciences, Northeastern University, Shenyang, China
}

Clinical studies have found that some Alzheimer's disease (AD) patients suffer from Cushing's syndrome (CS). CS is caused by the long-term release of excess glucocorticoids (GCs) from the adrenal gland, which in turn, impair brain function and induce dementia. Thus, we investigated the mechanism of the effect of corticosterone (CORT) on the development and progression of AD in a preclinical model. Specifically, the plasma CORT levels of 9-month-old APP/PS1 Tg mice were abnormally increased, suggesting an association between GCs and AD. Long-term administration of CORT accelerated cognitive dysfunction by increasing the production and deposition of $\beta$-amyloid (A $\beta$ ). The mechanism of action of CORT treatment involved stimulation of the expression of BACE-1 and presenilin (PS) 1 in in vitro and in vivo. This observation was confirmed in mice with adrenalectomy (ADX), which had lower levels of GCs. Moreover, the glucocorticoid receptor (GR) mediated the effects of CORT on the stimulation of the expression of BACE-1 and PS1 via the PKA and CREB pathways in neuroblastoma N2a cells. In addition to these mechanisms, CORT can induce a cognitive decline in APP/PS1 Tg mice by inducing apoptosis and decreasing the differentiation of neurons.

\footnotetext{
Keywords: Alzheimer's disease, corticosterone, glucocorticoid receptor, BACE-1, CREB, apoptosis,
} neuronal differentiation

\section{INTRODUCTION}

Clinical studies have found that Alzheimer's disease (AD) patients usually suffer from corticosteronism, which is characterized by the secretion of high levels of glucocorticoids (GCs) from the adrenal cortex (Guldiken and Guldiken, 2008; Haraguchi et al., 2016). In an A mouse model of an anxiety/depression-like state, long-term exposure to GCs results in structural changes in the brain, similar to those observed in aging (David et al., 2009; Herbert and Lucassen, 2016). In detail, the long-term administration of GCs can impair specific cognitive regions, such as the neocortex and hippocampus (Starkman et al., 2001). When the cortisol dropped to the basal level, the size of the hippocampus will be enlarged accompanied by improving the learning ability (Starkman et al., 2001, 2003). Reciprocally, chronic high levels of GC lead to a decrease in the volume of the hippocampus, which results in impairing cognitive ability (Starkman et al., 1992; Sheline et al., 1996; Bettio et al., 2017). More closely, patients with high levels of GC show long-term 
impairment of memory and concentration (Ragnarsson et al., 2012). Based on these prior works, GCs potentially contribute to accelerating the progression of $\mathrm{AD}$.

The production of GCs is tightly regulated under physiological conditions to avoid pathological effects. GCs is a self-regulating molecule in the endocrine system and is responsible for modulating stress reaction. GCs are synthesized and secreted by the fascicular zone of the adrenal cortex. GCs can be classified into two categories, hydrocortisone (HC) and cortisone, the synthesis and secretion of which are regulated by the hypothalamic-pituitary-adrenal (HPA) axis (Turnbull and Rivier, 1999). In detail, the nucleus of the paraventricular hypothalamus releases corticotropin-releasing factor (CRF), which promotes the secretion of adrenocorticotropic hormone $(\mathrm{ACTH})$ by acting on the anterior pituitary, leading to the synthesis of GCs by the fascicular zone of the posterior adrenal cortex (Curran and Chalasani, 2012). GCs is primarily synthesized and secreted from the adrenal cortex and penetrates the blood-brain barrier (BBB) to function in the central nervous system (CNS).

The physiological and pharmacological functions of GCs are mediated by the intracellular glucocorticoid receptor (GR), which belongs to the family of nuclear receptors. Thus, the GR can promote or inhibit the transcription of target genes by directly binding to the glucocorticoid response element (GRE) or interacting with other transcription factors (Ratman et al., 2013; Meijer et al., 2018). Mineralocorticoid receptors (MR) have higher GCs binding activity than the GR (Richardson et al., 2016; Faught and Vijayan, 2019). Under resting conditions, the level of GCs is relatively low and GCs mainly bind to MR. When the level of GCs is elevated under pathological conditions, GCs bind to and activate the GR to induce biological effects (Brinks et al., 2007). In AD patients, GR-expressing neurons undergo progressive atrophy and loss, resulting in a decrease in the expression of the GR, leading to the excessive loading of GCs by disrupting the negative feedback of the GR on the HPA axis that regulates GCs synthesis (Sapolsky et al., 1986; Jacobson and Sapolsky, 1991). Also, overexpression of the GR in male $\mathrm{C} 57 \mathrm{BL} / 6 \mathrm{~L}$ mice accelerates the aging phenotype, including neuroendocrine dysregulation and deficit in cognitive function (Wei et al., 2007). Specifically, blocking the GR for only 3 days in 12-month-old APP/PS1 Tg mice reduced the production of $A \beta_{1-40}$ and $A \beta_{1-42}$ in the hippocampus, resulting in the rescue of cognitive deficit (Lesuis et al., 2018). These observations were confirmed by reports that showed that treatment with a GR antagonist (mifepristone) completely reversed synaptic deficits and hippocampal apoptosis and partially reversed cognitive deficit, which are effects of the hippocampal amyloidogenic pathway and neuroinflammation (Pineau et al., 2016). Also, selective GR modulators can reverse hippocampal A $\beta$ generation, neuroinflammation, and apoptosis; restore the hippocampal levels of synaptic markers; and improve cognitive function (Pineau et al., 2016).

GCs have certain GR-independent effects on the regulation of $\mathrm{AD}$ development. For instance, administration of stresslevel GCs increases the formation of $A \beta$ by increasing the steady-state levels of amyloid precursor protein (APP) and
$\beta$-APP-cleaving enzyme (BACE-1) in APP/PS1/tau ${ }^{\mathrm{P} 301 \mathrm{~L}} \mathrm{Tg}$ mice (Green et al., 2006). This process is mediated by lipid raft-dependent CREB activation (Choi et al., 2017). Additionally, GCs considerably reduce the degradation and clearance of A $\beta$ by astrocytes, inducing a decrease in the neuroprotective ability of astrocytes (Wang et al., 2011). GCs act via these mechanisms to impair learning and memory by inducing apoptosis of neurons (Li et al., 2010).

Based on these considerations, we aimed to examine the multiple roles of GCs in the production and deposition of $A \beta$, neuronal apoptosis, and neuronal differentiation. The results demonstrate that GCs significantly increase the production of $A \beta$ by enhancing the expression of BACE-1 and PS1. Moreover, GCs induce neuronal apoptosis by reducing the ratio of $\mathrm{Bcl}-2$ and $\mathrm{Bax}$ and inhibiting neuronal differentiation. These effects eventually induce a cognitive decline in APP/PS1 Tg mice.

\section{MATERIALS AND METHODS}

\section{Reagents}

Corticosterone (CORT) was obtained from Solarbio Life Sciences (Beijing, China). A PKA inhibitor, H89; an antibody specific for A $\beta$ (A8354, mouse, dilution 1:100 for immunohistochemistry, IHC); and secondary antibodies were purchased from Sigma-Aldrich (St. Louis, MO, USA). Antibodies against APP (ab241592, rabbit, 1:2,000 for Western blot analysis), BACE1 (ab2077, rabbit, 1:2,000 for Western blot analysis; 1:100 for IHC and immunofluorescence, IF) and NeuN (ab104224, mouse, 1:100 for IF) were obtained from Abcam (Cambridge, MA, USA). Fluorescence-tagged secondary antibodies (A32727 mouse, A32732 rabbit, A11034 rabbit, A11029 mouse, 1:500 for IF) were purchased from Thermo Fisher Scientific (Waltham, MA, USA). Other antibodies, including ADAM10 (\#14194, rabbit, 1:2,000 for Western blot analysis), PS1 (\#5643, rabbit, 1:2,000 for Western blot analysis; 1:100 for IHC and IF), PS2 (\#2192, rabbit, 1:2,000 for Western blot analysis), PEN2 (\#5451, rabbit, 1:2,000 for Western blot analysis), nicastrin (\#5665, rabbit, 1:2,000 for Western blot analysis), GR (\#3660, rabbit, 1:2,000 for Western blot analysis; 1:100 for IF), CREB (\#4820, rabbit, 1:2,000 for Western blot analysis), p-CREB (\#9196, mouse, 1:2,000 for Western blot analysis), Bcl-2 (\#3498, rabbit, 1:2,000 for Western blot analysis), Bax (\#2774, rabbit, 1:2,000 for Western blot analysis), doublecortin (\#14802, rabbit, 1:100 for IHC) and $\beta$-actin (\#3700, mouse, 1:5,000 for Western blot analysis), were from Cell Signaling Technology (Danvers, MA, USA). All reagents for the sodium dodecyl sulfate-polyacrylamide gel electrophoresis (SDS-PAGE) experiments were purchased from Bio-Rad Laboratories (Hercules, CA, USA). All other reagents were from Thermo Fisher Scientific/Invitrogen (Waltham, MA, USA) unless specified otherwise.

\section{Cell Culture and Treatment}

Neuroblastoma N2a cells were cultured in DMEM with $10 \%$ FBS in a $37^{\circ} \mathrm{C}$ cell incubator containing $5 \% \mathrm{CO}_{2}$. Before treatment with corticosterone (CORT) or H89, the cells were cultured in a serum-free medium for $12 \mathrm{~h}$. After changing the 
serum-free medium, $1 \mu \mathrm{M}$ CORT or $10 \mu \mathrm{M}$ H89 was added to the fresh medium. After $24 \mathrm{~h}$, the cells were lysed to extract protein or RNA.

\section{Plasmid Construction}

The primers for gRNA generation were synthesized by Genewiz, Inc. (Suzhou, Jiangsu, China). The sequences of the primers were as follows: NR3C1-1 forward, 5'-CACCGGCTTTGGAT AAATCTGGCTG-3'; reverse, 5' -AAACCAGCCAGATTTATC CAAAGCC-3'; NR3C1-2 forward, 5' -CACCGGGATCATCTTC TCCCGCCAA- $3^{\prime}$; reverse, $5^{\prime}$-AAACTTGGCGGGAGAAGATG ATCCC-3'; and NR3C1-3 forward, 5'-CACCGCCAGCAGTT TGCTTGGCCGG-3' ; reverse, 5' -AAACCCGGCCAAGCAAAC TGCTGGC-3'. The paired forward and reverse primers were annealed in NE buffer 2.1 (New England Biolabs, Beverly, MA, USA). LentiCRISPR V2 plasmid (Addgene, Cambridge, MA, USA) was linearized by incubation at $37^{\circ} \mathrm{C}$ with BsmBI restriction enzymes for $2 \mathrm{~h}$. The annealed primers were used as insertion fragments, which were then inserted into the linearized LentiCRISPR v2 plasmid by incubating with T4 ligase at $16^{\circ} \mathrm{C}$ for $2 \mathrm{~h}$. The constructed plasmids were transferred to Stbl3 competent cells for amplification and subsequent Sanger sequencing.

\section{Transfection}

LentiCRISPR v2 plasmids ( $6 \mu \mathrm{g}$ ) encoding gRNA of NR3C1 were cotransfected with $5 \mu \mathrm{g}$ of psPAX2 and $3 \mu \mathrm{g}$ of pMD2.G into HEK293T cells using Neofect (Neofect Biotech Company Limited, Beijing, China) transfecting reagent. The medium was replaced after $12 \mathrm{~h}$. The virus was collected after 48 and $72 \mathrm{~h}$ of transfection by centrifugation at $8 \times 10^{4} \mathrm{rpm}$. The N2a cells were then infected with the virus in the presence of $10 \mu \mathrm{g} / \mathrm{ml}$ polybrene (Merck Millipore, Billerica, MA, USA). After $48 \mathrm{~h}$, the cells were selected in the presence of $3 \mu \mathrm{g} / \mathrm{ml}$ puromycin to establish NR3C1 knockdown cells.

\section{Animals and Treatment}

APP/PS1 Tg mice were obtained from Jackson Laboratory (Bar Harbor, ME, USA). C57BL/6J mice were purchased from the Liaoning Changsheng Biotechnology Company Limited (Benxi, Liaoning, China). All mice were housed under the same conditions at standard room temperature and humidity in a light and dark cycle-controlled environment. To ensure that mice could freely move, eat, and drink, three to five mice were housed per cage. APP/PS1 Tg mice were used as a model for the AD study. The WT or APP/PS1 Tg mice at the age of 5 months were subcutaneously administered with CORT (10 mg/kg/day) for 3 months before assessment of their memory and physiological and biochemical parameters; the timeline and group sorting are shown in Figures 1A,B.

\section{Adrenalectomy}

The WT or APP/PS1 Tg mice were fasted overnight before surgery and anesthetized by tribromoethanol (Sigma-Aldrich, Beijing, China). Mice were fixed in a prone position, and the long hair from the middle of the back was removed. A $1.5 \mathrm{~cm}$ midline incision was made, and muscle was exfoliated layer by layer from the outside to the inside until the organs were visible.
The spherical adrenal tissue around the kidney was identified and gently removed with scissors, which was then placed back in the body before closing the wound. The other side of the adrenal gland was removed using the same method. The sham operation group only received an incision, and the skin was sutured. From the 3rd day after adrenalectomy (ADX) surgery, intraperitoneal injection of CORT $(10 \mathrm{mg} / \mathrm{kg} /$ day $)$ was performed for 7 days. Then, tribromoethanol was used for anesthesia, and the animals were sacrificed to collect the cerebral cortex and hippocampus.

\section{qRT-PCR}

Total RNA was extracted by RNA isolator total RNA extraction reagent (Nanjing Vazyme Medical Technology Company Limited, Nanjing, Jiangsu, China). Purified RNA was reversetranscribed into cDNA by a reverse transcription kit (Promega, Madison, WI, USA). qRT-PCR was performed according to the instructions of the qRT-PCR kit (Promega, Madison, WI, USA). The primer sequences for $\mathrm{qRT}-\mathrm{PCR}$ were as follows: ADAM10 forward, 5'-CTCAAGCTTCGAAT TCATGGTG TTGCCGACAGTGTT-3'， reverse， 5'-GCGACCGGTGGATC CTTGCGTCGCATGTGTCCCAT-3'; BACE1 forward, 5'-CT CAAGCTTCGAATTCCAAGGCCCGGGCTCACTATG-3', reverse, 5'-GGCGACCGGTGGATCCGCCTTGAGCAGGGA GATGTCATCA-3' PS1 forward, 5'-CTCAAGCTTCGAATTC CTCCAATGACAGAGATACCTG-3', reverse, 5' -GGCGACCG GTGGATCCGCGATATAAAACTGATGGAATG-3'; GAPDH forward, 5' -TGCAGTGGCAAAGTGGAGAT-3' , reverse, 5' -TT $^{\prime}$ TGCCGTGAGTGGAGTCATA-3'. GAPDH was used as a housekeeping reference gene. The ratio was calculated as the following equation:

$$
\text { Ratio }=\frac{2^{\Delta C t\left(\mathrm{Gene}_{\mathrm{wt}}-\mathrm{Gene}_{\mathrm{Tg}}\right)}}{2^{\Delta C t\left(\mathrm{GAPDH}_{\mathrm{wt}}-\mathrm{GAPDH}_{\mathrm{Tg}}\right)}}
$$

The WT was always set to 1 , and the value of the Tg mice was obtained from the previous equation.

\section{Western Blots}

Cells or tissues of the cerebral cortex and hippocampus were homogenized and lysed on ice in RIPA buffer [ $25 \mathrm{mM}$ Tris- $\mathrm{HCl}$ (Ph 7.6), $150 \mathrm{mM} \mathrm{NaCl}, 1 \% \mathrm{NP}-40,1 \%$ sodium deoxycholate, and $0.1 \%$ SDS], containing protease inhibitor cocktail (Thermo Scientific-Pierce, Rockford, IL, USA). The supernatant was collected after centrifugation at $13,000 \mathrm{~g}$. The concentration of protein in the supernatant was determined by a BCA kit (Beyotime Biotechnology, Shanghai, China). Protein $(2 \mu \mathrm{g} / \mu \mathrm{l})$ was loaded onto SDS-PAGE gels and, following SDS-PAGE, was transferred to a PVDF membrane. The membranes were incubated with a primary antibody at $4^{\circ} \mathrm{C}$ overnight. The membranes were washed with TBST five times for 5 min each time. Then, the corresponding secondary antibody was added to the membranes and incubated at room temperature for $2 \mathrm{~h}$. The membranes were probed with an antibody, and the bands were visualized by an ECL kit (Thermo Fisher Scientific, Waltham, MA, USA). $\beta$-Actin was used as an internal reference control. 

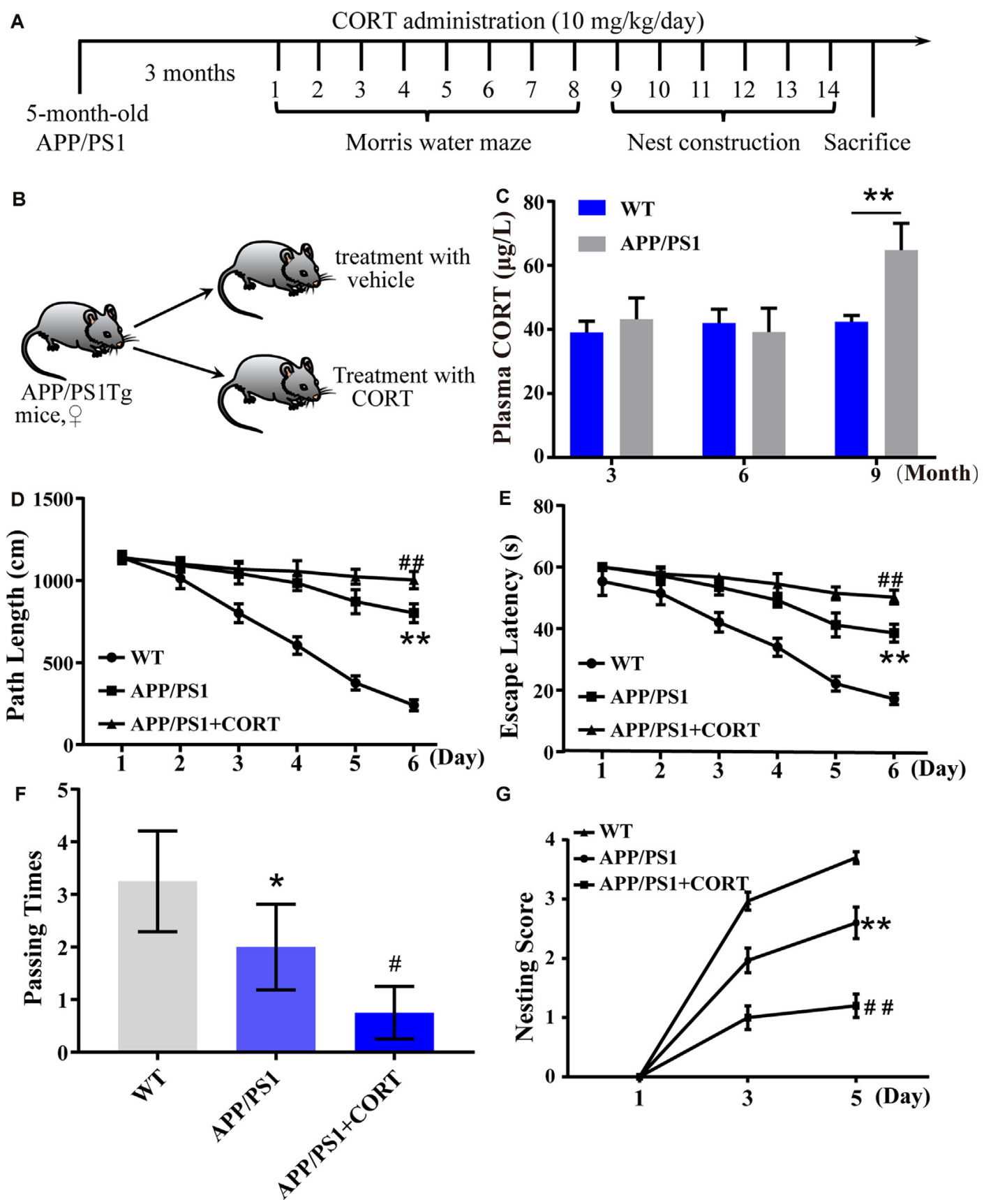

G

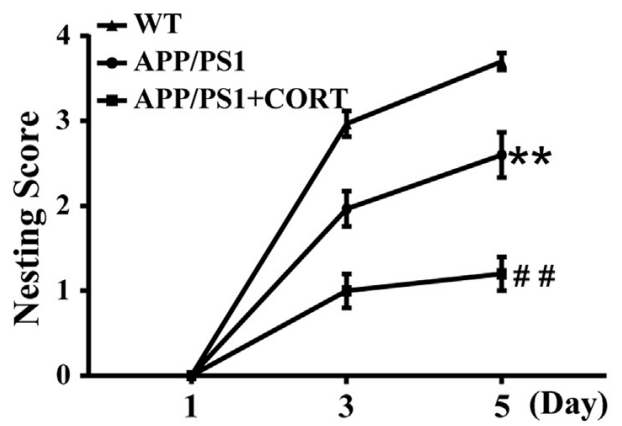

FIGURE 1 | Elevated plasma levels of corticosterone (CORT) result in impaired learning in APP/PS1 Tg mice. (A) Timeline of the treatment of APP/PS1 Tg mice with CORT. (B) Schematic diagram of the random grouping and treatment of mice. (C) Plasma was collected from 3-, 6- and 9-month-old APP/PS1 Tg and age-matched WT mice. The content of CORT was determined by enzyme-linked immunosorbent assay (ELISA). The data were analyzed by repeated-measures ANOVA $(n=6)$. (D,E) APP/PS1 Tg mice (5-month-old) were intraperitoneally injected with CORT (10 mg/kg/day) for 3 months. (D,E) The escape distance and latency of mice were observed in the hidden platform experiment. (F) In the space exploration experiment, the crossing times to the original platform were recorded by the software. The data were analyzed by repeated-measures ANOVA ( $n=6)$. (G) The nesting score of various groups of mice was analyzed by the nest construction assay. The data were analyzed by a nonparametric statistical test. The data are presented as the mean \pm SE of independent experiments $(n=6)$. ${ }^{*} p<0.05 ;{ }^{* *} p<0.01$ compared with the WT mice. ${ }^{\#} p<0.05$; ${ }^{\# \#} p<0.01$ compared with vehicle-treated APP/PS1 Tg mice.

\section{Immunohistochemistry (IHC)}

The brains of WT or APP/PS1 Tg mice were fixed with $4 \%$ paraformaldehyde and dehydrated in a $30 \%$ sucrose solution. Sections ( $30 \mu \mathrm{m}$ thickness) were prepared by a push slicer (Leica,
Wetzlar, Germany). The slides were submerged in $3 \%$ hydrogen peroxide, to eliminate endogenous peroxidase activity. The sections were stained with antibodies specific for $A \beta$, BACE1, PS1, or doublecortin at $4^{\circ} \mathrm{C}$ overnight. A biotin-conjugated 
secondary antibody was then incubated with the sections for 30 min at room temperature after rinsing three times with PBS. The sections were then stained using a DAB solution [7 mg DAB $+50 \mathrm{ml}$ Tris- $\mathrm{HCl}(\mathrm{pH} \mathrm{7.6})+12 \mu \mathrm{H}_{2} \mathrm{O}_{2}$ ]. Before mounting, the slides were dehydrated with graded ethanol and were washed with xylene for $20 \mathrm{~min}$. Finally, the sections were observed and imaged under an optical microscope (Leica, Wetzlar, Germany) using a $10 \times$ or $20 \times$ objective lens.

\section{Immunofluorescence}

The cells or cerebral cortex and hippocampus tissue samples were fixed with $4 \%$ paraformaldehyde, permeabilized with $50 \mu \mathrm{g} / \mathrm{ml}$ digitonin for $10 \mathrm{~min}$, and washed with PBS three times. The slides were blocked with $4 \%$ goat serum and incubated with a primary antibody specific to NeuN or GR at $4^{\circ} \mathrm{C}$ overnight. Then, the sections were incubated with Alexa 555-anti-rabbit IgG (1:500), Alexa 488-anti-rabbit IgG (1:500), and DAPI $(1: 1,000)$ for $1 \mathrm{~h}$ at room temperature. After rinsing, the sections were mounted using a fluorescent mounting reagent (Beyotime Biotechnology, Shanghai, China). The sections were visualized and imaged under a confocal microscope (Leica, Wetzlar, Germany) equipped with a $40 \times$ or $63 \times$ oil-immersion objective lens.

\section{Enzyme-Linked Immunosorbent Assay (ELISA)}

The production of $\mathrm{A} \beta_{1-42}$ was determined by enzyme-linked immunosorbent assay (ELISA) kits (Thermo Fisher Scientific, Waltham, MA, USA). In brief, $50 \mu \mathrm{l}$ of the standard curve storage solution and the sample solution were added to 96-well plates. Then, an $A \beta_{1-42}$ antibody was added to the plate. After rinsing with wash buffer, the HRP-conjugated secondary antibody was incubated with the samples. After another rinse, the stabilized chromogen TMB was added to the plate for $30 \mathrm{~min}$ before measurement at $450 \mathrm{~nm}$ in a microplate reader.

CORT was assayed by ELISA kits (Wuhan Enzyme ImmunoBiotechnology Company Limited, Wuhan, Hubei, China) according to the manufacturer's protocol. Briefly, $50 \mu \mathrm{l}$ of the standard curve storage solution and the sample solution were added to 96-well plates. Enzyme-labeled reagent $(50 \mu \mathrm{l})$ was added to the plates, which were incubated at $37^{\circ} \mathrm{C}$ for $30 \mathrm{~min}$. After washing, $50 \mu \mathrm{l}$ of developer $\mathrm{A}$ and developer B were added to the wells. After a $10 \mathrm{~min}$ incubation at $37^{\circ} \mathrm{C}, 50$ $\mu l$ stop solution was added to the wells before determination of the optical density of the CORT signal at $450 \mathrm{~nm}$ in a microplate reader.

\section{Morris-Maze Test}

APP/PS1 Tg mice at the age of 5 months were treated with CORT (10 mg/kg/day) for 3 months. The Morris maze test was used to determine spatial learning and memory. In brief, mice were placed into a $1.6 \mathrm{~m}$ diameter pool, which had labeled quadrants, I, II, III, and IV. Milk was added to the pool, and the water was then heated to $25 \pm 2{ }^{\circ} \mathrm{C}$. In the first 2 days, the platform was set $1-2 \mathrm{~cm}$ above the water level in quadrant II. The time and distance required for mice to find the platform was recorded for $60 \mathrm{~s}$. If a mouse was unable to find the platform in $60 \mathrm{~s}$, the mouse was guided to the platform or placed on the platform and kept on the platform for an additional $10 \mathrm{~s}$. Starting from day 3, the platform was submerged under the water. The time and distance required for mice to find the platform was recorded in the following 4 days. On day 7, the platform was removed, and the number of crosses to the original location of the platform was recorded for $60 \mathrm{~s}$. The experimental timeline is shown in Figure 1A.

\section{Nest Construction}

After the Morris water maze test, each mouse was housed in a separate cage. Ten pieces of five $5 \mathrm{~cm}^{2}$ filter paper were placed in the cage. Each mouse was imaged for 6 days at the same time every day. The specific evaluation rules were as follows: 0 , no nesting behavior of mice and no tearing or moving of paper; 1 , no obvious nesting behavior in mice, and only slight tearing and moving of paper; 2, no nesting behavior in mice, and obvious tearing and moving of paper; 3 , nesting behavior in mice, and most of the paper was torn into wicker shapes; and 4, obvious nesting behavior in mice, and all the paper was torn and moved to a corner. Finally, the mice were anesthetized and sacrificed using tribromoethanol to collect the cerebral cortex and hippocampus.

\section{Statistical Analysis}

All data are presented as the mean $\pm \mathrm{SE}$, of at least three independent experiments. Data from the Morris water maze test and detection of CORT in plasma were analyzed using repeatedmeasures ANOVA. The paired $t$-test was used to analyze the nest construction data. Statistical significance of the differences between means was determined using Student's $t$-test or one-way analysis of variance when appropriate.

\section{RESULTS}

\section{The Levels of CORT Are Elevated in the Plasma of APP/PS1 Tg Mice}

In patients with sporadic $\mathrm{AD}$, the plasma level of cortisol is increased in the early and late stages of the disease (Umegaki et al., 2000). The secretion of GCs is regulated by the HPA axis (Turnbull and Rivier, 1999). However, it is not known whether HPA axis dysfunction is the initial cause of AD. To detect whether the basic level of GCs is changed in AD animal models and whether the metabolic pathway of amyloid can change the production of GCs, the plasma CORT level of APP/PS1 Tg mice of various ages was detected. According to the data provided by Jackson Laboratory, APP/PS1 Tg mice express chimeric Mo/HuAPP695swe and mutant PS1-dE9 genes, $\mathrm{A} \beta$ is deposited in the brain of 6-month-old mice. Based on this information, plasma samples of wild-type (WT) and Tg mice were collected to determine the levels of CORT at the ages of 3, 6, and 9 months. The results demonstrated that the plasma levels in 3- and 6-month-old APP/PS1 animals were not significantly different from those of WT mice. In contrast, the plasma levels of 9-month-old APP/PS1 Tg mice were higher than those of the corresponding WT controls (Figure 1C). This observation suggests that enhanced production of GCs is associated with amyloidosis. 


\section{CORT Exposure Accelerates the Cognitive Decline of APP/PS1 Tg Mice}

To determine whether CORT can promote the pathological process in $\mathrm{AD}$ model mice, 5-month-old APP/PS1 Tg mice were intraperitoneally injected with CORT at a dose of $10 \mathrm{mg} / \mathrm{kg} / \mathrm{day}$ for 3 months. The Morris maze test was performed to determine the learning and memory ability of mice. The results showed that mice treated with GCs take more time and travel a longer distance to find the hidden platform compared to untreated APP/PS1 Tg mice (Figures 1D,E). In the space exploration experiment, the crossing times of GCs-treated mice were considerably lower than those of vehicle-treated controls (Figure 1F), suggesting that GCs impairs the leaning ability of APP/PS1 Tg mice.

The nest-building test is an experimental method to assess the social ability of mice. The results can reflect the instinctive learning and social ability of mice based on biting paper and constructing a nest. In the nesting experiment, the nesting ability of mice treated with CORT for 3 months was significantly decreased compared with that of vehicle-treated controls (Figure 1G). In conclusion, CORT can accelerate the decline in learning and memory ability in mice, suggesting that a high level of GCs can accelerate the occurrence and development of $\mathrm{AD}$ in vivo.

\section{CORT Treatment Enhances the Deposition of $A \beta$ in the Brain of APP/PS1 Tg Mice}

According to $A \beta$ theory, abnormal production and deposition of $A B$ in the APs of the cerebral cortex and hippocampus is the key cause of the onset of AD (O'Brien and Wong, 2011). Thus, the effects of CORT on the production and deposition of $\mathrm{A} \beta$ in APP/PS1 Tg mice were assessed. APP/PS1 Tg mice (5-month-old) were intraperitoneally injected with CORT at a dose of $10 \mathrm{mg} / \mathrm{kg} / \mathrm{day}$ for 3 months. The formation of APs was determined by IHC in CORT-treated APP/PS1 Tg mice. The results showed that the number of APs in the cerebral cortex and hippocampus of APP/PS1 Tg mice was increased by CORT treatment compared to that in vehicle-treated controls (Figures 2A,B). The plasma concentration of CORT was determined by ELISA, and the results showed that treatment with a high dose of CORT elevated the plasma levels of CORT in APP/PS1 Tg mice (Figure 2C). Moreover, the production of $A \beta_{1-42}$ was determined by ELISA. The results showed that the production of $A \beta_{1-42}$ in the cerebral cortex and hippocampus of APP/PS1 Tg mice was stimulated by long-term administration of CORT (Figure 2D). Therefore, these results demonstrate that GCs can enhance the production and deposition of $A \beta$ in APP/PS1 Tg mice.

\section{CORT Increases the Production of $A \beta$ by Enhancing the Expression of BACE-1 and PS1}

In the amyloid metabolic pathway, APP is sequentially cleaved by $\beta$ - and $\gamma$-secretases to produce $A \beta$ (O'Brien and Wong, 2011). To determine the mechanisms of $A \beta$ production, the effects of CORT on the expression of $\alpha$-, $\beta$ - and $\gamma$-secretases was estimated. The results showed that CORT $(1 \mu \mathrm{M})$ treatment increases the expression of the BACE1 and PS1 proteins in N2a cells (Figures $3 A, B$ ). In agreement with the results of the Western blot analysis, qRT-PCR data showed that CORT treatment upregulated the expression of BACE1 and PS1 mRNA in N2a cells (Figure 3C). The immunofluorescent imaging results confirmed the upregulation of BACE1 and PS1 in N2a cells (Figures 3D,E). Considering the in vitro observations, the ability of GCs to upregulate the expression of BACE1 and PS1 in vivo was tested. According to Western blot analysis, CORT treatment increased the expression of the BACE1, PS1, and protein level of $\beta$-CTF, while reduced the level of $\alpha$-CTF without altering the protein level of APP in APP/PS1 Tg mice (Figures 3F-H). To assess the expression and distribution of BACE1 and PS1 in the brain of APP/PS1 Tg mice, sections were immunostained with antibodies against BACE1 and PS1. The results showed that the expression of BACE1 and PS1 was significantly increased in the $\mathrm{CA} 3$ region of the hippocampus (Figures 3I,J). Thus, CORT can promote the production of $\mathrm{A} \beta$ by activating BACE1 and PS1 in APP/PS1 Tg mice.

To investigate the role of CORT in the upregulation of the expression of BACE1 and PS1, WT, and APP/PS1 Tg mice were subjected to adrenalectomy (ADX) to establish a GCs-deficient animal model. After recovery for 3 days, ADX mice were randomly divided into vehicle and CORT $(10 \mathrm{mg} / \mathrm{kg} /$ day) groups and treated for 7 days. The level of CORT in plasma was determined by ELISA. The results demonstrated that ADX lowered the plasma level of CORT in WT and APP/PS1 Tg mice, which was restored by treatment of WT mice with CORT (Figures 4A,D). Protein expression of BACE1 and PS1 was always consistent with the level of CORT (Figures $4 \mathrm{~B}, \mathrm{C}, \mathrm{E}, \mathrm{F}$ ), suggesting the key role of CORT in the regulation of the expression of BACE1 and PS1 in mice.

\section{GR Mediates the Effects of CORT on Stimulating the Expression of BACE1 and PS1}

According to the classical hypothesis of the GCs cascade, the dysfunction of the HPA axis induced by a decrease in the GR level is the initial cause of AD. Thus, the expression of the GR was determined in CORT-treated mice. The results demonstrated that treatment with CORT $(10 \mathrm{mg} / \mathrm{kg} /$ day $)$ for 3 months did not significantly lower the expression of the GR (Figures 5A,B). However, the effects of the GR on the regulation of the expression of BACE1 and PS1 cannot be neglected. To assess these effects, the distribution of the GR was determined by immunofluorescence staining. The results demonstrated that the GR colocalized with neurons (Figure 5C). Therefore, the expression of NR3C1, which encodes the GR in N2a cells (Sevilla and Pérez, 2018) was knocked down and the efficacy of knocking down was determined by Western blots. The results demonstrated that gRNA targeted NR3C1 efficiently decreased the protein levels of NR3C1. The expression of BACE1 and PS1 was attenuated by knocking down the expression of NR3C1 in CORT-treated N2a cells (Figures 5D,E). Thus, the GR mediates the effects of GCs on stimulating the expression of BACE1 and PS1 in neurons. 

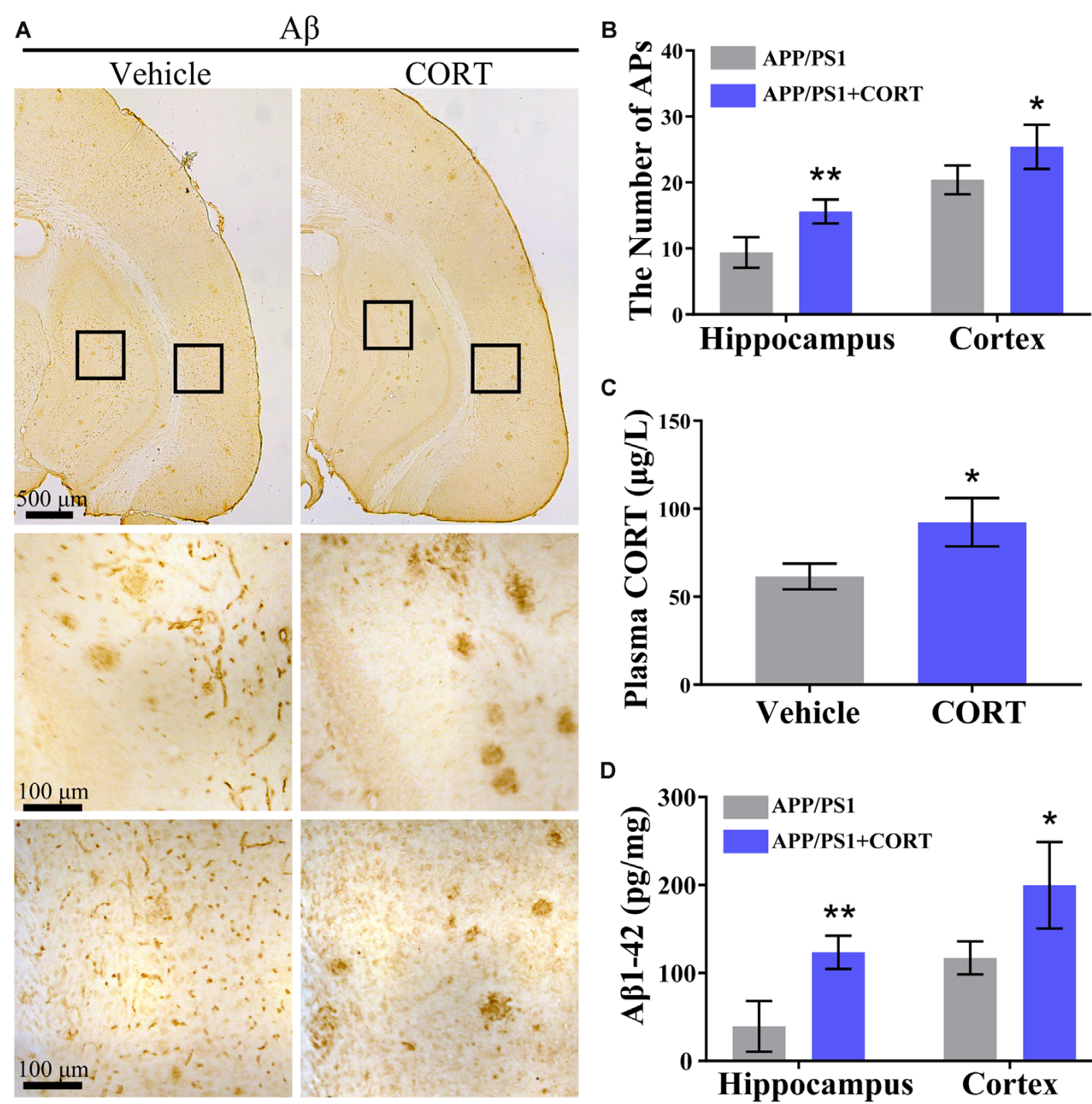

D

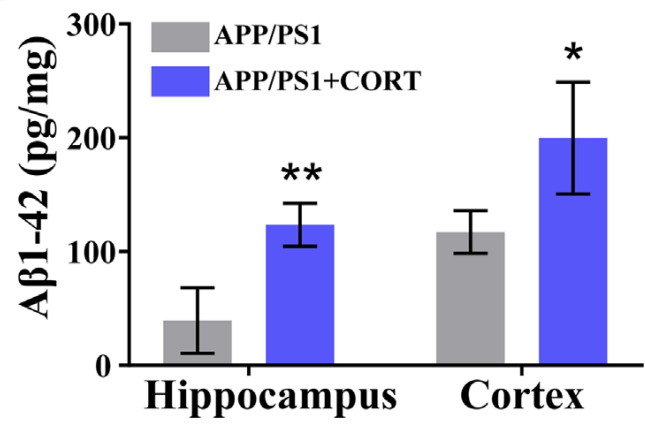

FIGURE 2 | CORT treatment increases the production of A $\beta$ and the number of APs in the brain of APP/PS1 Tg mice. APP/PS1 Tg mice (5-month-old) were intraperitoneally injected with CORT (10 mg/ $\mathrm{kg} /$ day) for 3 months. (A,B) The distribution of APs in the cerebral cortex and hippocampus of APP/PS1 Tg mice was determined by immunohistochemistry (IHC). (C) The content of CORT was determined by ELISA. (D) The contents of A $\beta_{1-42}$ in the cerebral cortex and hippocampus of APP/PS1 Tg mice were detected by ELISA. The data are present as the mean \pm SE of independent experiments $(n=6)$. ${ }^{*} p<0.05 ;{ }^{* *} p<0.01$ compared with the vehicle-treated mice by $t$-test.

\section{CORT Upregulates the Expression of BACE1 and PS1 via the PKA and CREB Signaling Pathways}

CREB is an important molecule involved in the regulation of memory in $\mathrm{AD}$ and was thus included in the current study to determine the mechanisms of its effects (Bartolotti and Lazarov, 2019). APP/PS1 mice were intraperitoneally injected with CORT at a dose of $10 \mathrm{mg} / \mathrm{kg} /$ day for 3 months. The Western blot analysis results indicated that phosphorylation of CREB was elevated in CORT-treated APP/PS1 Tg mice (Figures 6A,B). To validate these observations, $\mathrm{N} 2 \mathrm{a}$ cells were treated with CORT $(1 \mu \mathrm{M})$ in the absence or presence of a PKA/CREB inhibitor, H89 (10 $\mu \mathrm{M})$. After
$24 \mathrm{~h}$, phosphorylation of CREB was blocked by $\mathrm{H} 89$ in CORT-stimulated N2a cells (Figures 6C,D). Deactivation of CREB resulted in attenuation of the effects of CORT on the stimulation of the expression of BACE1 and PS1 (Figures 6C,D). These results suggest the PKA and CREB signaling pathways are involved in mediating the effects of CORT on the upregulation of the expression of BACE1 and PS1.

\section{CORT Promotes Apoptosis of Neurons and Inhibits Neuronal Differentiation in the Brain of APP/PS1 Tg Mice}

Our results identified the mechanisms of action of CORT in the regulation of the production and deposition of $A \beta$; thus, the 


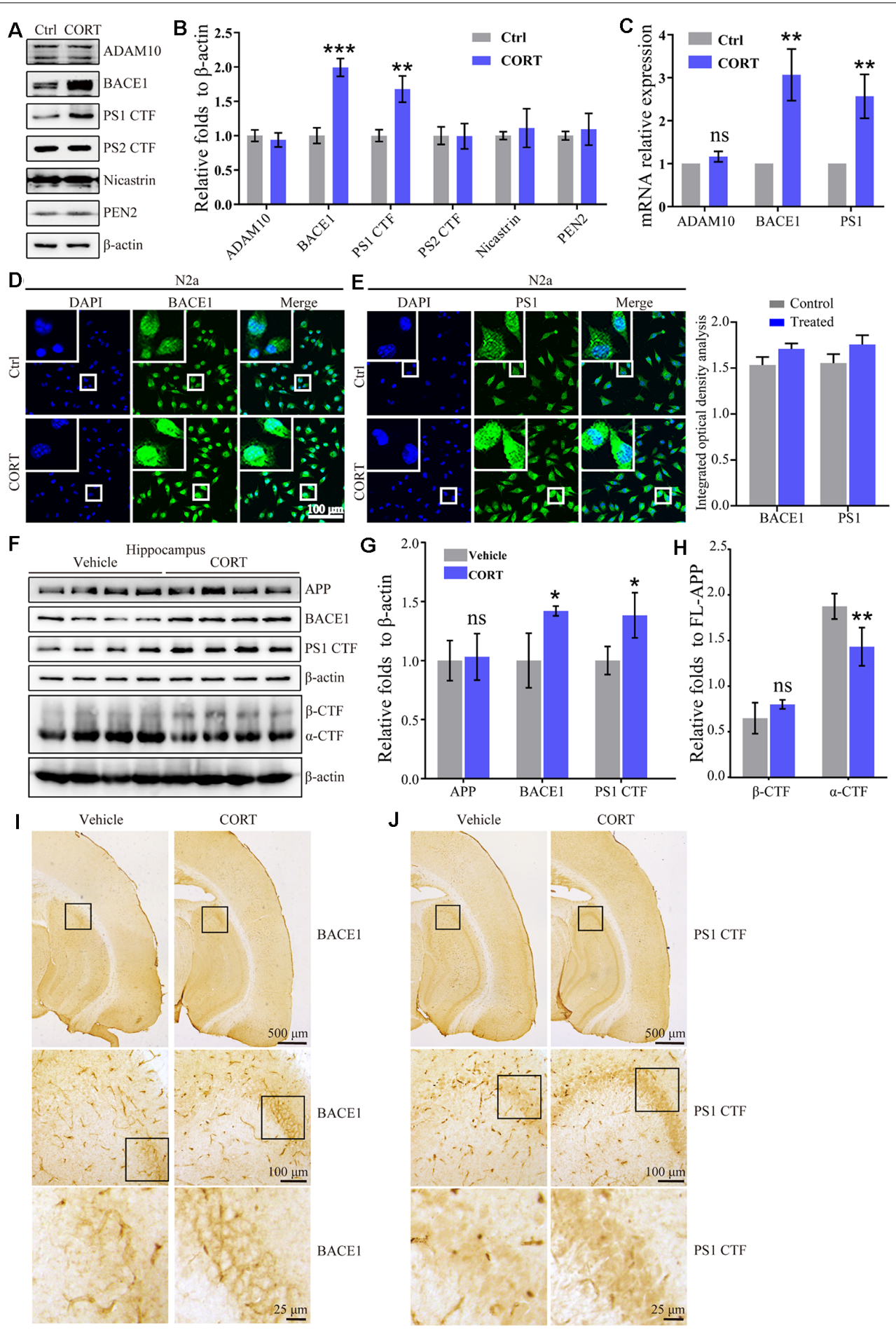

FIGURE 3 | CORT treatment upregulates the expression of BACE1 and PS1 in vitro and in vivo. (A-E) N2a cells were treated with CORT (1 $\mu$ M) for 24 h. (A) Western blot analysis was used to detect the expression of ADAM10, BACE1, PS1, PS2, nicastrin, and PEN2. $\beta$-actin was used as an internal control. (B) The optical density of the bands was analyzed by ImageJ. The data of gene expression were analyzed by the $t$-test $(n=6)$. (C) mRNA expression of ADMA10, BACE1, and PS1 was determined by qRT-PCR. GAPDH was used as a housekeeping gene. The gene expression data were analyzed by the $t$-test $(n=6)$. ${ }^{* \star} p<0.01$; ${ }^{\star \star \star} p<0.001$; ns: no significance compared with the vehicle-treated N2a cells. (D,E) The distribution of BACE1 and PS1 was detected by immunofluorescence and the immunofluorescence was semi-quantitatively analyzed by ImageJ software. (F-I) APP/PS1 Tg mice (5-month-old) were intraperitoneally injected with CORT (10 mg/kg/day) for 3 months. (F-H) The protein levels of APP, BACE1, PS1 $\alpha$-CTF, and $\beta$-CTF were determined by Western blot analysis. $\beta$-Actin was used as an internal control. The optical density of the bands was analyzed by ImageJ. The gene expression data were analyzed by the $t$-test $(n=6)$. (I,J) The distribution of BACE1 and PS1 was determined by IHC. The data are presented as the mean \pm SE of independent experiments. ${ }^{\star} p<0.05 ;{ }^{* *} p<0.01 ;{ }^{* * *} p<0.001$ compared with the vehicle-treated mice. 


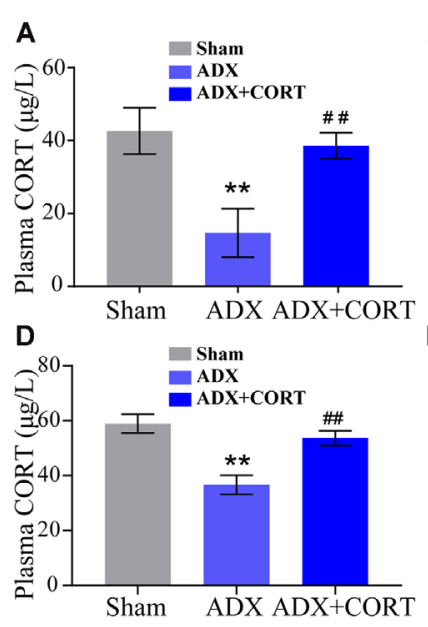

B

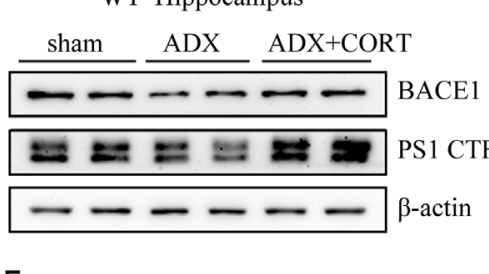

E

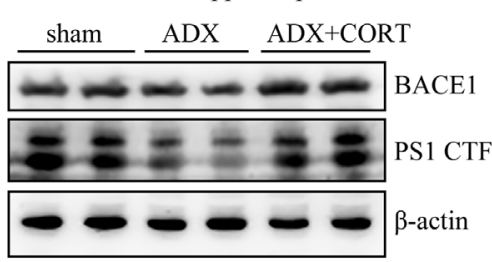

C

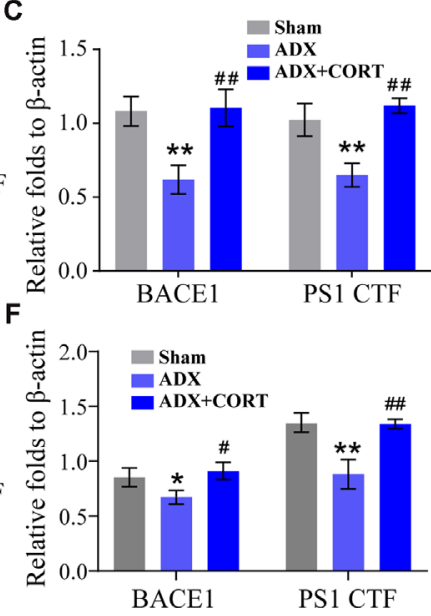

FIGURE 4 | CORT is essential for the expression of BACE1 and PS1. (A-C) WT mice were subjected to adrenalectomy (ADX). After 3 days, mice were intraperitoneally injected with CORT (10 mg/kg/day) for 7 days. (A) The content of CORT was determined by ELISA. The data were analyzed by repeated-measures ANOVA $(n=4)$. (B,C) Protein expression of BACE1 and PS1 was determined by Western blot analysis. $\beta$-Actin was used as an internal control. The optical density of the bands was analyzed by ImageJ. The gene expression data were analyzed by one-way ANOVA $(n=4) .{ }^{* *} p<0.01$ compared with the sham-operated mice. \#\# $p<0.01$ compared with the ADX-operated C57BL/6 mice. (D-F) APP/PS1 mice were subjected to adrenalectomy (ADX). After 3 days, mice were intraperitoneally injected with CORT (10 mg/kg/day) for 7 days. (D) The content of CORT was determined by ELISA. The data were analyzed by repeated-measures ANOVA ( $n=4)$. (E,F) Protein expression of BACE1 and PS1 was determined by Western blot analysis. $\beta$-Actin was used as an internal control. The optical density of the bands was analyzed by ImageJ. The gene expression data were analyzed by one-way ANOVA $(n=4)$. The data are presented as the mean \pm SE of independent experiments. ${ }^{\star} p<0.05 ;{ }^{* *} p<0.01$ compared with the sham-operated mice. ${ }^{\#} p<0.05,{ }^{\# \#} p<0.01$ compared with the ADX-operated APP/PS1 Tg mice.

roles of CORT in neurons were further investigated. A series of studies have suggested the neurotoxic effects of $A \beta$ on apoptosis of neurons (Reddy and Beal, 2008; Calvo-Rodriguez et al., 2019). To determine the effect of CORT on the brain of APP/PS1 Tg mice and to assess whether CORT can promote apoptosis of neurons in the brain, Western blot analysis was used to detect the expression of Bcl-2 and Bax in the hippocampus in all groups of mice. The results showed that the ratio of Bcl-2 to Bax was significantly downregulated in the brain of CORT-treated APP/PS1 Tg mice compared with that in the control groups (Figures 7A,B). This observation suggests that CORT promotes apoptosis of neurons in the brain of APP/PS1 transgenic mice. Since CORT induces apoptosis of neurons, the roles of CORT in neuronal differentiation were determined by staining doublecortin. The results demonstrated that axons and dendrites generated from neuronal stem cells are suppressed by treatment with CORT (Figure 7C). These results indicate that CORT can aggravate AD via multiple mechanisms, such as induction of the production and deposition of $A \beta$, triggering apoptosis of neurons, and inhibiting neuronal differentiation.

\section{DISCUSSION}

As the involvement of GCs in regulating the learning and cognitive functions (Starkman et al., 2001, 2003), we investigated the effects of GCs on $\mathrm{AD}$. As a consequence, we found that the content of CORT in plasma was significantly increased in the 9-month-old APP/PS1 Tg mice. By subcutaneously injected CORT, the learning ability of APP/PS1 Tg mice was impaired with enhancing the production and deposition of $\mathrm{A}$ in a
BACE1- and PS1-dependent mechanism. To the mechanism, CORT activates BACE1 and PS1 via the glucocorticoid receptor (GR)-PKA-CREB signaling pathway. These observations were further confirmed by ADX treatment, which downregulates the expression of BACE1 and PS1 by reducing the plasma levels of CORT.

Prior studies have shown that stress can accelerate brain aging, which results in the induction of dementia (Brinks et al., 2007). Additionally, stress has an impact on the major depressive disorder (MDD; Roy et al., 2017). Accumulating evidence shows that patients with MDD have cognitive disorders with symptoms similar to AD patients (Boedeker et al., 2020). These observations define a potential link between stress, MDD, and AD (Galts et al., 2019). Specifically, stress, MDD, and $\mathrm{AD}$ share the same characteristic of overproduction of GCs. Long-term exposure to a normal concentration of GCs accelerates brain aging, which results in a loss of the ability to regulate the levels of GCs, thus leading to further acceleration of brain aging and, eventually, causing brain impairment and onset of dementia. However, it is unclear whether GCs leads to the onset and development of $\mathrm{AD}$ or plays a role in $\mathrm{AD}$-associated mechanisms. Certain controversies regarding the specific molecular mechanisms require additional clarification despite numerous studies on these issues. Therefore, the roles of CORT in the regulation of the development and progression of $\mathrm{AD}$ were investigated in the present study. The results indicate that CORT has multiple pathological functions in the progression of $\mathrm{AD}$, including regulation of the production and aggregation of $A \beta$, apoptosis of neurons and suppression of neuronal differentiation. 
A

Hippocampus

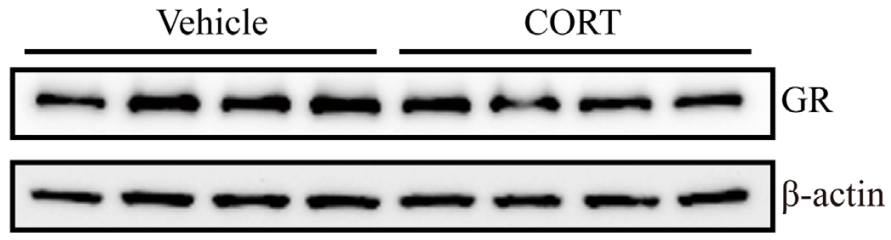

C
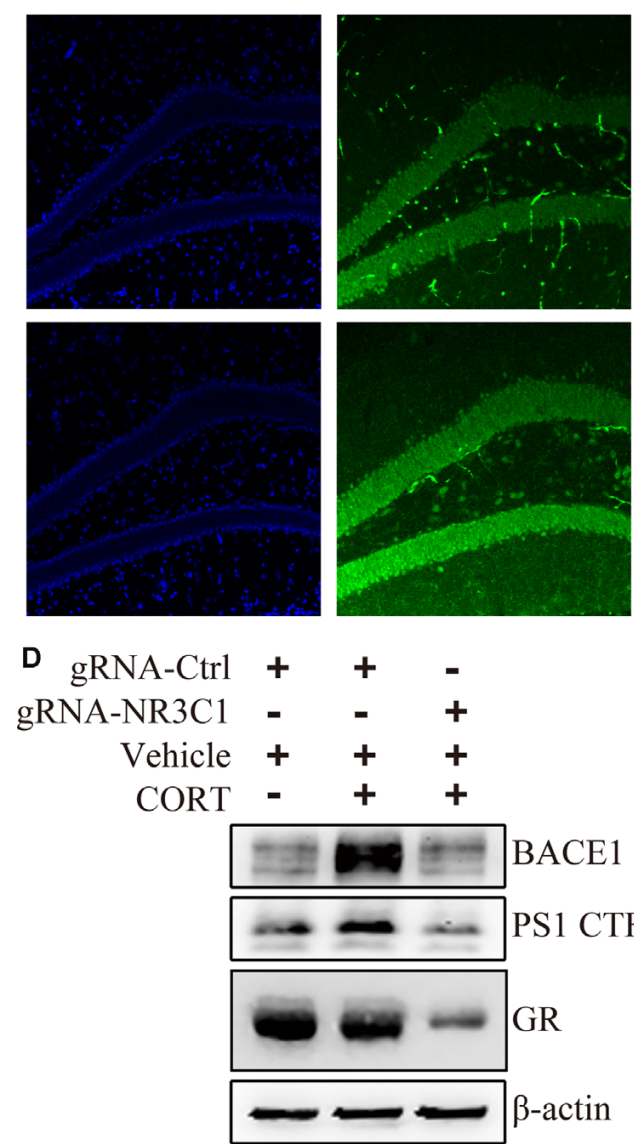

NeuN
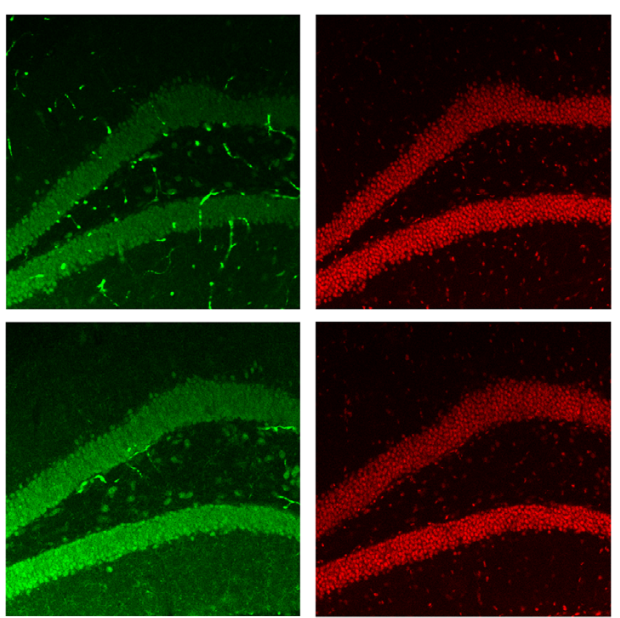

E

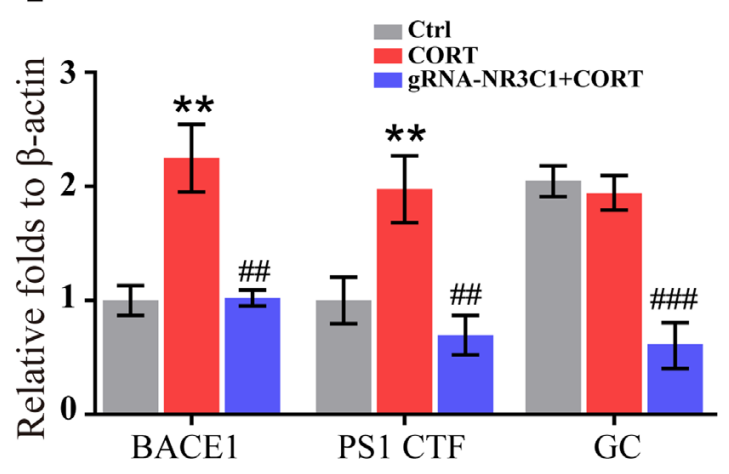

FIGURE 5 | Glucocorticoid receptor (GR) mediates the effects of CORT on the upregulation of the expression of BACE1 and PS1 in neurons. (A-C) APP/PS1 Tg mice (5-month-old) were intraperitoneally injected with CORT (10 mg/kg/day) for 3 months. (A,B) The expression of the GR was determined by Western blot analysis. $\beta$-Actin was used as an internal control. The optical density of the bands was analyzed by ImageJ. The data were analyzed by the $t$-test $(n=6)$. ns: no significance compared with the vehicle-treated controls. (C) Brain sections of mice were double-stained with NeuN and GR antibodies and observed by confocal microscopy. (D,E) N2a cells were treated with CORT with or without knockdown of NR3C1 expression. Western blot analysis was used to detect the expression of BACE1, PS1, and NR3C1. $\beta$-Actin was used as an internal control. The optical density of the bands was analyzed by ImageJ. The data were analyzed by one-way ANOVA $(n=6)$. The data are presented as the mean \pm SE of independent experiments. ${ }^{* *} p<0.01$ compared with the vehicle-treated controls. ${ }^{\# \#} p<0.01$; $\# \#$ \#\# 0.001 compared with the CORT-treated N2a cells.

Clinical data show that the average daily fluctuations of cortisol levels in elderly people are higher than those in younger people (Raskind et al., 1994). In the last century, elevated plasma levels of cortisol have been reported to induce a reduction in the volume of the hippocampus and impairment of memory in elderly people. Certain patients with high cortisol levels progressively develop AD (Lupien et al., 2005). Similarly, GCs are also responsible for a decrease in the volume of the hippocampus and memory impairment in patients with Cushing's syndrome (CS; Forget et al., 2000). 

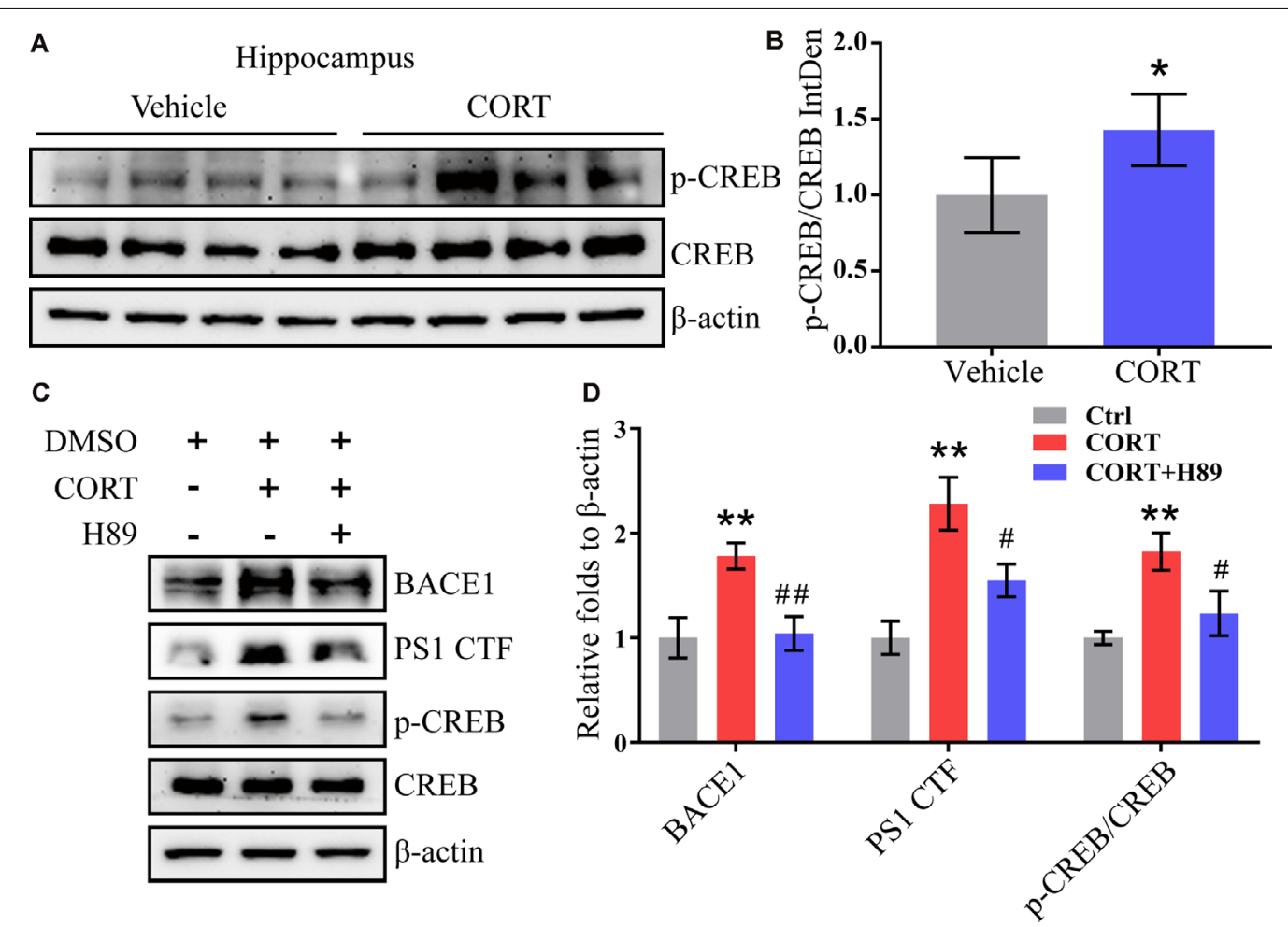

FIGURE 6 | CORT upregulates the expression of BACE1 and PS1 via the PKA and CREB signaling pathway. (A,B) APP/PS1 Tg mice (5-month-old) were intraperitoneally injected with CORT (10 mg/kg/day) for 3 months. The levels of phosphorylated and total CREB were determined by Western blot analysis. $\beta$-Actin was used as an internal control. The ratio of $p$-CREB to CREB was calculated using ImageJ software. The data were analyzed by the $t$-test $(n=6)$. (C,D) N2a cells were treated with CORT $(1 \mu \mathrm{M})$ in the absence or presence of H89 $(10 \mu \mathrm{M})$ for $24 \mathrm{~h}$. The expression levels of BACE-1, PS1, p-CREB, and CREB were measured by Western blot analysis. $\beta$-Actin was used as an internal control. The optical density was measured using Image J software. The gene expression data were analyzed by one-way ANOVA $(n=6)$. The data are presented as the mean \pm SE of independent experiments. ${ }^{*} p<0.05$; ${ }^{* *} p<0.01$ compared with the vehicle-treated controls. ${ }^{\#} p<0.05$; ${ }^{\#} p<0.01$ compared with CORT-treated N2a cells.

In agreement with these observations, the level of cortisol is significantly higher in $\mathrm{AD}$ patients than in the control subjects (Raskind et al., 1982). Also, epidemiological studies have revealed that high levels of cortisol can upregulate the expression of $\mathrm{AD}$-associated genes and can be used to diagnose $\mathrm{AD}$ via long-term monitoring of the level of cortisol (Ennis et al., 2017). The plasma concentration of CORT in $3 \times \mathrm{Tg} \mathrm{AD}$ mice was significantly higher than that in WT mice (Green et al., 2006). Thus, the prior studies have been expanded and it has been demonstrated that the plasma concentration of CORT is elevated in 9-month-old APP/PS1 Tg mice compared to that in WT mice.

However, overloading of GCs is not always bad for brain aging. For example, acute and transient secretion of GCs is beneficial to some types of learning and memory, such as emotional ability (McGaugh and Roozendaal, 2002) and spatial memory (Wingenfeld and Wolf, 2014). In contrast, a series of studies have demonstrated that GCs can increase the levels of $\mathrm{Ca}^{2+}$-mediated electrophysiological biomarkers of hippocampal aging in the range from a few minutes to several hours (Kerr et al., 1989, 1992; Joëls and de Kloet, 1993). Therefore, the effects of short-term exposure to GCs on learning and memory remain a matter of debate. However, high levels of GCs or long-term exposure to a normal concentration of GCs will continuously activate the GR, which results in damage to memory (Zhang et al., 2020). In a preliminary study in the salmon and mammalian (including human) brain, an increase in GCs resulted in the degeneration of peripheral tissues, similar to the changes associated with aging (Sotiropoulos et al., 2008). In an ADX group of rats, several biomarkers of brain aging were significantly reduced, resulting in improved learning and memory performance in the Morris maze test compared with that in the sham group (Landfield et al., 1981). GCs have been shown to damage the hippocampus by increasing its vulnerability (Sapolsky et al., 1986). In detail, GCs impairs the energy metabolism of hippocampal neurons by inhibiting the uptake of glucose, which results in an increase in the susceptibility of cultured neurons to all types of destructive metabolic damage (Sapolsky et al., 1988). Additionally, CORT defines the relationship between hippocampal damage and cognitive impairment in elderly rats (Issa et al., 1990; Meaney et al., 1995), which is supported by other reports that demonstrated a negative correlation between GCs exposure and cognitive decline. On the other hand, excessive loading with GCs will cause atrophy of hippocampal dendrites and delay neurogenesis (McEwen, 1996; Mirescu and Gould, 2006). Based 


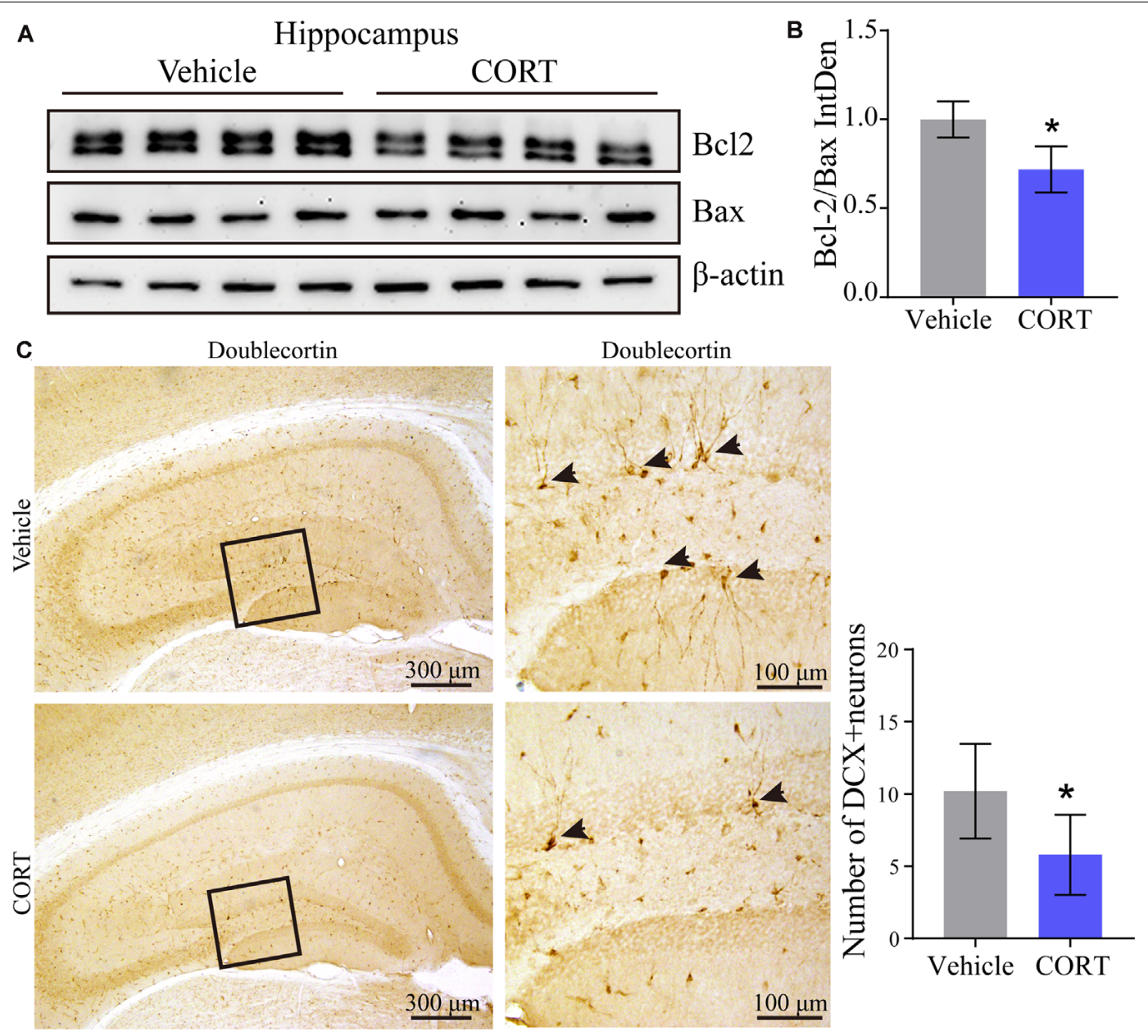

FIGURE 7 | CORT promotes apoptosis of neurons and inhibits neuronal differentiation in the brain of APP/PS1 Tg mice. APP/PS1 Tg mice (5-month-old) were intraperitoneally injected with CORT (10 mg/kg/day) for 3 months. (A,B) Western blot analysis was used to detect the expression of Bcl-2 and Bax in the hippocampus of mice in each group. $\beta$-Actin was used as an internal control. The bands were analyzed using ImageJ software. The data were analyzed by the $t$-test $(n=6)$. (C) Neuronal differentiation was determined by IHC staining of doublecortin. The positive neurons were semi-quantitatively analyzed by Image s software. The data are presented as the mean \pm SE of independent experiments. ${ }^{*} p<0.05$ compared with the vehicle-treated controls.

on these studies, CORT treatment-induced cognitive decline in APP/PS1 Tg mice.

Secretion of GCs is regulated by the HPA axis. The GR is the key molecule of the HPA axis that regulates the level of GCs in vivo. Under pathological conditions, the concentrations of GCs increases in vivo. High levels of GCs act on the GR in the hippocampus, hypothalamus, and pituitary, which in turn, induce a decrease in GCs to the basal level via a negative feedback mechanism. Release of GCs leads to downregulation of the expression of the GR and its downstream signaling pathway and atrophy and loss of GR-containing neurons in the hippocampus, which suppresses the regulatory effects of the GR on the HPA axis (Sotiropoulos et al., 2008). The mRNA and protein levels of the GR are decreased with age in the hippocampus, damaging the negative feedback regulation of GCs and leading to continuous exposure of the brain to high concentrations of GCs, which results in loss of neuronal function (Landfield, 1978;
Landfield et al., 1978; Jacobson and Sapolsky, 1991). Without chronic GCs stress, overexpression of the GR in the mouse forebrain has been reported to be responsible for the acceleration of the expression of brain aging-like phenotype (Wei et al., 2007). Also, subchronic inhibition of the GR in AD model mice can treat early defects of situational memory and synaptic plasticity (Lanté et al., 2015). The GR is a nuclear receptor that acts as a transcription factor to mediate the effects of CORT on the upregulated expression of BACE1 and PS1. GCs bind to the GR in the cytoplasm, which then enters the nucleus to regulate the transcription of APP and BACE1 via binding to the CRE sequence of the APP and BACE1 promoters (Green et al., 2006). The present study expands on these previous findings by demonstrating that knockdown of expression of NR3C1 blocks the effects of CORT on stimulating the expression of BACE1 and PS2, suggesting that CORT upregulates the expression of BACE1 and PS1 via the GR in neurons. 
CREB is a transcription factor that plays an important role in regulating the transition from short-term memory to long-term memory (Tully et al., 2003; Saura and Valero, 2011). Additionally, the CREB signal is involved in the regulation of processes associated with many neurodegenerative diseases, such as $\mathrm{AD}$. For example, $\mathrm{CREB}$ has been reported to regulate the expression of BACE1 and PS1 as a transcription factor (Lahiri et al., 2006; Choi et al., 2017). The results of the present study demonstrated that CORT can stimulate the phosphorylation of CREB at Ser 133, which results in upregulation of the expression of BACE1 and PS1 in neurons. The addition of H89 completely blocked the effects of CORT on stimulating the expression of BACE1 and PS1 via dephosphorylation of $\mathrm{CREB}$, suggesting the key role of $\mathrm{CREB}$ in mediating the effects of CORT on regulating the expression of BACE1 and PS1 in neurons.

According to A $\beta$ theory, APP is abnormally cleaved to produce $A \beta$, which can induce apoptosis of neurons via multiple pathways (Reddy and Beal, 2008; Calvo-Rodriguez et al., 2019). $\mathrm{A} \beta$ can concurrently upregulate the expression of Bax and downregulate the expression of Bcl-2, thus disrupting the balance between Bax and Bcl-2, leading to apoptosis of neurons during the course of $\mathrm{AD}$ development and progression ( $\mathrm{Hu}$ et al., 2016). The results of the present study indicate that CORT can induce the production of $A \beta$ and decrease the ratio of Bcl-2 to Bax, suggesting that CORT promotes apoptosis of neurons. Moreover, CORT inhibits neuronal differentiation in APP/PS1 Tg mice. In agreement with these results, chronic exposure to high concentrations of CORT has been shown to reduce neuronal differentiation in the dentate gyrus of rats (Brummelte and Galea, 2010). Inhibition of the secretion of CORT from midlife to the rest of the animal life increases neuronal differentiation in old animals, which prevents the onset of age-related memory disorders (Montaron et al., 2006). Thus, the effects of CORT trigger a loss of neurons during AD development and progression.

For neuroinflammation, more and more data proved the crosstalk between neuroinflammation and $A \beta$, which can accelerate the progression of AD (Cai et al., 2014). Since studies have shown that GCs induce the expression of nod-like receptor family in cultured macrophages and primary macrophages, leading to the secretion of pro-inflammatory cytokines IL-1, TNF- $\alpha$, and IL-6 (Busillo et al., 2011), we continue to detect if CORT can regulate the neuroinflammation of APP/PS1 Tg mice. As the main cell types that mediate neuroinflammation in the brain are astrocytes and microglia (Sawikr et al., 2017), we immunostained the glial cells with GFAP and Iba1. The results demonstrated that CORT activates the glial cells of APP/PS1 Tg mice, leading to the secretion of TNF- $\alpha$ (data not shown). In agreement with our results, CORT has been reported to activate microglial cells (Zalewska et al., 2017) and astrocytes (Bridges et al., 2008). Moreover, intravenous infusion of CORT at non-stressed (35 ng/ml) and stressed levels (350 ng/ml) increased the release of TNF- $\alpha$ and/or IL-6 in the liver (Liao et al., 1995).

Apart from the above effects, high GCs can inhibit mTOR-dependent autophagy, leading to the aggregation and deposition of tau, which results in neuronal death of $\mathrm{Tau}^{\mathrm{P} 301 \mathrm{~L}}$ mice (Silva et al., 2019). Also, GCs in the brain are the key regulators of dendritic spines. For example, Pedrazzoli et al. (2019) found that dexamethasone, an agonist of GRs, can significantly reduce the density of dendritic spines in the hippocampal CA1 area of 6- and 10-month-old 3× Tg-AD mice. Moreover, recent work suggests that GCs may regulate synaptic plasticity by interacting with glutamate-energy mechanisms and ultimately affect learning and memory processes (Sandi, 2011).

\section{CONCLUSIONS}

Elevated levels of CORT during the course of $\mathrm{AD}$ development and progression can induce the production and deposition of $\mathrm{A} \beta$ in APs by activating BACE1 and PS1 in APP/PS1 Tg mice. Additionally, the GR mediates the effects of high dose CORT exposure by stimulating the expression of BACE1 and PS1 via the PKA and CREB signaling cascades. These mechanisms of CORT promote apoptosis of neurons and inhibit neuronal differentiation, resulting in cognitive decline in APP/PS1 Tg mice.

\section{DATA AVAILABILITY STATEMENT}

The raw data supporting the conclusions of this article will be made available by the authors, without undue reservation.

\section{ETHICS STATEMENT}

The animal study was reviewed and approved by medical laboratory animals (Ministry of Health, Peoples Republic of China, 1998) and the laboratory animal ethical standards of Northeastern University of China were adhered to.

\section{AUTHOR CONTRIBUTIONS}

S-QZ and L-LC conceived and performed all of the experiments, participated in the design of the study, and wrote the manuscript. Y-YL carried out select experiments. PW interpreted the data and wrote the manuscript. All authors contributed to the article and approved the submitted version.

\section{FUNDING}

This work was supported, in part or in whole, by the National Natural Science Foundation of China $(\mathrm{CN} ; 81870840)$ and the Fundamental Research Funds for the Central Universities, China (N172008008).

\section{SUPPLEMENTARY MATERIAL}

The Supplementary Material for this article can be found online at: https://www.frontiersin.org/articles/10.3389/fnmol.2020.61 3421/full\#supplementary-material. 


\section{REFERENCES}

Bartolotti, N., and Lazarov, O. (2019). CREB signals as PBMC-based biomarkers of cognitive dysfunction: a novel perspective of the brainimmune axis. Brain Behav. Immun. 78, 9-20. doi: 10.1016/j.bbi.2019. 01.004

Bettio, L. E. B., Rajendran, L., and Gil-Mohapel, J. (2017). The effects of aging in the hippocampus and cognitive decline. Neurosci. Biobehav. Rev. 79, 66-86. doi: 10.1016/j.neubiorev.2017.04.030

Boedeker, S., Schulz, P., Beblo, T., Lenz, E., Sammer, G., Kreisel, S., et al. (2020). Symbol comprehension in patients with Alzheimer disease dementia, mild cognitive impairment and major depressive disorder. Alzheimer Dis. Assoc. Disord. 34, 85-93. doi: 10.1097/WAD.000000000 0000347

Bridges, N., Slais, K., and Syková, E. (2008). The effects of chronic corticosterone on hippocampal astrocyte numbers: a comparison of male and female Wistar rats. Acta Neurobiol. Exp. 68, 131-138.

Brinks, V., van der Mark, M. H., de Kloet, E. R., and Oitzl, M. S. (2007). Differential MR/GR activation in mice results in emotional states beneficial or impairing for cognition. Neural Plast. 2007:90163. doi: 10.1155/2007/90163

Brummelte, S., and Galea, L. A. (2010). Chronic high corticosterone reduces neurogenesis in the dentate gyrus of adult male and female rats. Neuroscience 168, 680-690. doi: 10.1016/j.neuroscience.2010.04.023

Busillo, J. M., Azzam, K. M., and Cidlowski, J. A. (2011). Glucocorticoids sensitize the innate immune system through regulation of the NLRP3 inflammasome. J. Biol. Chem. 286, 38703-38713. doi: 10.1074/jbc.M111.275370

Cai, Z., Hussain, M. D., and Yan, L. J. (2014). Microglia, neuroinflammation and $\beta$-amyloid protein in Alzheimer's disease. Int. J. Neurosci. 124, 307-321. doi: 10.3109/00207454.2013.833510

Calvo-Rodriguez, M., Hernando-Perez, E., Nuñez, L., and Villalobos, C. (2019). Amyloid $\beta$ oligomers increase ER-mitochondria $\mathrm{Ca}^{2+}$ cross talk in young hippocampal neurons and exacerbate aging-induced intracellular $\mathrm{Ca}^{2+}$ remodeling. Front. Cell Neurosci. 13:22. doi: 10.3389/fncel.2019. 00022

Choi, G. E., Lee, S. J., Lee, H. J., Ko, S. H., Chae, C. W., and Han, H. J. (2017). Membrane-associated effects of glucocorticoid on BACE1 upregulation and $A \beta$ generation: involvement of lipid raft-mediated CREB activation. J. Neurosci. 37, 8459-8476. doi: 10.1523/JNEUROSCI.0074-17.2017

Curran, K. P., and Chalasani, S. H. (2012). Serotonin circuits and anxiety: what can invertebrates teach us? Invert. Neurosci. 12, 81-92. doi: 10.1007/s10158012-0140-y

David, D. J., Samuels, B. A., Rainer, Q., Wang, J. W., Marsteller, D., Mendez, I., et al. (2009). Neurogenesis-dependent and -independent effects of fluoxetine in an animal model of anxiety/depression. Neuron 62, 479-493. doi: 10.1016/j. neuron.2009.04.017

Ennis, G. E., An, Y., Resnick, S. M., Ferrucci, L., O’Brien, R. J., and Moffat, S. D. (2017). Long-term cortisol measures predict Alzheimer disease risk. Neurology 88, 371-378. doi: 10.1212/WNL.0000000000003537

Faught, E., and Vijayan, M. M. (2019). Postnatal triglyceride accumulation is regulated by mineralocorticoid receptor activation under basal and stress conditions. J. Physiol. 597, 4927-4941. doi: 10.1113/JP278088

Forget, H., Lacroix, A., Somma, M., and Cohen, H. (2000). Cognitive decline in patients with Cushing's syndrome. J. Int. Neuropsychol. Soc. 6, 20-29. doi: 10.1017/s1355617700611037

Galts, C. P. C., Bettio, L. E. B., Jewett, D. C., Yang, C. C., Brocardo, P. S., Rodrigues, A. L. S., et al. (2019). Depression in neurodegenerative diseases: common mechanisms and current treatment options. Neurosci. Biobehav. Rev. 102, 56-84. doi: 10.1016/j.neubiorev.2019.04.002

Green, K. N., Billings, L. M., Roozendaal, B., McGaugh, J. L., and LaFerla, F. M. (2006). Glucocorticoids increase amyloid- $\beta$ and tau pathology in a mouse model of Alzheimer's disease. J. Neurosci. 26, 9047-9056. doi: 10.1523/JNEUROSCI.2797-06.2006

Guldiken, S., and Guldiken, B. (2008). Subclinical Cushing's syndrome is a potential cause of metabolic dementia and rapidly progressive Alzheimer-type dementia. Med. Hypotheses 71, 703-705. doi: 10.1016/j.mehy.2008.05.036

Haraguchi, Y., Mizoguchi, Y., Noguchi, T., Arai, T., Fukuyama, J., Kato, T. A., et al. (2016). A patient with Alzheimer's disease complicated by elderlyonset Cushing's syndrome who had undergone surgical treatment for adrenocorticotropic hormone-independent macronodular adrenal hyperplasia. Psychogeriatrics 16, 274-276. doi: 10.1111/psyg.12146

Herbert, J., and Lucassen, P. J. (2016). Depression as a risk factor for Alzheimer's disease: genes, steroids, cytokines and neurogenesis-what do we need to know? Front. Neuroendocrinol. 41, 153-171. doi: 10.1016/j.yfrne.2015. 12.001

Hu, H., Wang, Y., Zhang, Y., Wang, W., Xu, D., Chen, Z., et al. (2016). Effect of Qingxinkaiqiao compound on cortical mRNA expression of the apoptosisrelated genes Bcl-2, BAX, caspase- 3 and A $\beta$ in an Alzheimer's disease rat model. J. Tradit. Chin. Med. 36, 654-662. doi: 10.1016/s0254-6272(16)30086-3

Issa, A. M., Rowe, W., Gauthier, S., and Meaney, M. J. (1990). Hypothalamicpituitary-adrenal activity in aged, cognitively impaired and cognitively unimpaired rats. J. Neurosci. 10, 3247-3254. doi: 10.1523/JNEUROSCI.10-1003247.1990

Jacobson, L., and Sapolsky, R. (1991). The role of the hippocampus in feedback regulation of the hypothalamic-pituitary-adrenocortical axis. Endocr. Rev. 12, 118-134. doi: 10.1210/edrv-12-2-118

Joëls, M., and de Kloet, E. R. (1993). Corticosteroid actions on amino acid-mediated transmission in rat CA1 hippocampal cells. J. Neurosci. 13, 4082-4090. doi: 10.1021/acsami.0c18454

Kerr, D. S., Campbell, L. W., Hao, S. Y., and Landfield, P. W. (1989). Corticosteroid modulation of hippocampal potentials: increased effect with aging. Science 245, 1505-1509. doi: 10.1126/science.2781293

Kerr, D. S., Campbell, L. W., Thibault, O., and Landfield, P. W. (1992). Hippocampal glucocorticoid receptor activation enhances voltage-dependent $\mathrm{Ca}^{2+}$ conductances: relevance to brain aging. Proc. Natl. Acad. Sci. U S A 89, 8527-8531. doi: 10.1073/pnas.89.18.8527

Lahiri, D. K., Ge, Y. W., Rogers, J. T., Sambamurti, K., Greig, N. H., and Maloney, B. (2006). Taking down the unindicted co-conspirators of amyloid $\beta$-peptide-mediated neuronal death: shared gene regulation of BACE1 and APP genes interacting with CREB, Fe65 and YY1 transcription factors. Curr. Alzheimer Res. 3, 475-483. doi: 10.2174/156720506779025224

Landfield, P. W. (1978). An endocrine hypothesis of brain aging and studies on brain-endocrine correlations and monosynaptic neurophysiology during aging. Adv. Exp. Med. Biol. 113, 179-199. doi: 10.1007/978-1-4684-8893-7_11

Landfield, P. W., Baskin, R. K., and Pitler, T. A. (1981). Brain aging correlates: retardation by hormonal-pharmacological treatments. Science 214, 581-584. doi: $10.1126 /$ science. 6270791

Landfield, P. W., Waymire, J. C., and Lynch, G. (1978). Hippocampal aging and adrenocorticoids: quantitative correlations. Science 202, 1098-1102. doi: $10.1126 /$ science.715460

Lanté, F., Chafai, M., Raymond, E. F., Pereira, A. R., Mouska, X., Kootar, S., et al. (2015). Subchronic glucocorticoid receptor inhibition rescues early episodic memory and synaptic plasticity deficits in a mouse model of Alzheimer's disease. Neuropsychopharmacology 40, 1772-1781. doi: 10.1371/journal.pone. 0242588

Lesuis, S. L., Weggen, S., Baches, S., Lucassen, P. J., and Krugers, H. J. (2018). Targeting glucocorticoid receptors prevents the effects of early life stress on amyloid pathology and cognitive performance in APP/PS1 mice. Transl. Psychiatry 8:53. doi: 10.1038/s41398-018-0101-2

Li, W. Z., Li, W. P., Yao, Y. Y., Zhang, W., Yin, Y. Y., Wu, G. C., et al. (2010). Glucocorticoids increase impairments in learning and memory due to elevated amyloid precursor protein expression and neuronal apoptosis in 12-month old mice. Eur. J. Pharmacol. 628, 108-115. doi: 10.1016/j.ejphar.2009. 11.045

Liao, J., Keiser, J. A., Scales, W. E., Kunkel, S. L., and Kluger, M. J. (1995). Role of corticosterone in TNF and IL-6 production in isolated perfused rat liver. Am. J. Physiol. 268, R699-R706. doi: 10.1152/ajpregu.1995.268. 3.R699

Lupien, S. J., Schwartz, G., Ng, Y. K., Fiocco, A., Wan, N., Pruessner, J. C., et al. (2005). The douglas hospital longitudinal study of normal and pathological aging: summary of findings. J. Psychiatry Neurosci. 30, 328-334.

McEwen, B. S. (1996). Gonadal and adrenal steroids regulate neurochemical and structural plasticity of the hippocampus via cellular mechanisms involving NMDA receptors. Cell. Mol. Neurobiol. 16, 103-116. doi: 10.1007/BF02088170

McGaugh, J. L., and Roozendaal, B. (2002). Role of adrenal stress hormones in forming lasting memories in the brain. Curr. Opin. Neurobiol. 12, 205-210. doi: $10.1016 /$ s0959-4388(02)00306-9 
Meaney, M. J., O’Donnell, D., Rowe, W., Tannenbaum, B., Steverman, A., Walker, M., et al. (1995). Individual differences in hypothalamic-pituitaryadrenal activity in later life and hippocampal aging. Exp. Gerontol. 30, 229-251. doi: 10.1016/0531-5565(94)00065-b

Meijer, O. C., Koorneef, L. L., and Kroon, J. (2018). Glucocorticoid receptor modulators. Ann. Endocrinol. 79, 107-111. doi: 10.1016/j.ando.2018.03.004

Mirescu, C., and Gould, E. (2006). Stress and adult neurogenesis. Hippocampus 16, 233-238. doi: 10.1002/hipo.20155

Montaron, M. F., Drapeau, E., Dupret, D., Kitchener, P., Aurousseau, C., Le Moal, M., et al. (2006). Lifelong corticosterone level determines age-related decline in neurogenesis and memory. Neurobiol. Aging 27, 645-654. doi: 10.1016/j.neurobiolaging.2005.02.014

O’Brien, R. J., and Wong, P. C. (2011). Amyloid precursor protein processing and Alzheimer's disease. Annu. Rev. Neurosci. 34, 185-204. doi: 10.1146/annurevneuro-061010-113613

Pedrazzoli, M., Losurdo, M., Paolone, G., Medelin, M., Jaupaj, L., Cisterna, B., et al. (2019). Glucocorticoid receptors modulate dendritic spine plasticity and microglia activity in an animal model of Alzheimer's disease. Neurobiol. Dis. 132:104568. doi: 10.1016/j.nbd.2019.104568

Pineau, F., Canet, G., Desrumaux, C., Hunt, H., Chevallier, N., Ollivier, M., et al. (2016). New selective glucocorticoid receptor modulators reverse amyloid- $\beta$ peptide-induced hippocampus toxicity. Neurobiol. Aging 45, 109-122. doi: 10.1016/j.neurobiolaging.2016.05.018

Ragnarsson, O., Berglund, P., Eder, D. N., and Johannsson, G. (2012). Long-term cognitive impairments and attentional deficits in patients with Cushing's disease and cortisol-producing adrenal adenoma in remission. J. Clin. Endocrinol. Metab. 97, E1640-E1648. doi: 10.1210/jc.2012 $-1945$

Raskind, M., Peskind, E., Rivard, M. F., Veith, R., and Barnes, R. (1982). Dexamethasone suppression test and cortisol circadian rhythm in primary degenerative dementia. Am. J. Psychiatry 139, 1468-1471. doi: 10.1176/ajp.139. 11.1468

Raskind, M. A., Peskind, E. R., and Wilkinson, C. W. (1994). Hypothalamicpituitary-adrenal axis regulation and human aging. Ann. N. Y. Acad. Sci. 746, 327-335. doi: 10.1111/j.1749-6632.1994.tb39251.x

Ratman, D., Vanden Berghe, W., Dejager, L., Libert, C., Tavernier, J., Beck, I. M., et al. (2013). How glucocorticoid receptors modulate the activity of other transcription factors: a scope beyond tethering. Mol. Cell. Endocrinol. 380, 41-54. doi: 10.1016/j.mce.2012.12.014

Reddy, P. H., and Beal, M. F. (2008). Amyloid $\beta$, mitochondrial dysfunction and synaptic damage: implications for cognitive decline in aging and Alzheimer's disease. Trends Mol. Med. 14, 45-53. doi: 10.1016/j.molmed.2007. 12.002

Richardson, R. V., Batchen, E. J., Denvir, M. A., Gray, G. A., and Chapman, K. E. (2016). Cardiac GR and MR: from development to pathology. Trends Endocrinol. Metab. 27, 35-43. doi: 10.1016/j.tem.2015. 10.001

Roy, B., Shelton, R. C., and Dwivedi, Y. (2017). DNA methylation and expression of stress related genes in PBMC of MDD patients with and without serious suicidal ideation. J. Psychiatr. Res. 89, 115-124. doi: 10.1016/j.jpsychires.2017. 02.005

Sandi, C. (2011). Glucocorticoids act on glutamatergic pathways to affect memory processes. Trends Neurosci. 34, 165-176. doi: 10.1016/j.tins.2011.01.006

Sapolsky, R. M., Krey, L. C., and McEwen, B. S. (1986). The neuroendocrinology of stress and aging: the glucocorticoid cascade hypothesis. Endocr. Rev. 7, 284-301. doi: 10.1210/edrv-7-3-284

Sapolsky, R. M., Packan, D. R., and Vale, W. W. (1988). Glucocorticoid toxicity in the hippocampus: in vitro demonstration. Brain Res. 453, 367-371. doi: 10.1016/0006-8993(88)90180-1

Saura, C. A., and Valero, J. (2011). The role of CREB signaling in Alzheimer's disease and other cognitive disorders. Rev. Neurosci. 22, 153-169. doi: 10.1515/RNS.2011.018

Sawikr, Y., Yarla, N. S., Peluso, I., Kamal, M. A., Aliev, G., and Bishayee, A. (2017). Neuroinflammation in Alzheimer's disease: the preventive and therapeutic potential of polyphenolic nutraceuticals. Adv. Protein Chem. Struct. Biol. 108, 33-57. doi: 10.1016/bs.apcsb.2017.02.001
Sevilla, L. M., and Pérez, P. (2018). Roles of the glucocorticoid and mineralocorticoid receptors in skin pathophysiology. Int. J. Mol. Sci. 19:1906. doi: 10.3390/ijms19071906

Sheline, Y. I., Wang, P. W., Gado, M. H., Csernansky, J. G., and Vannier, M. W. (1996). Hippocampal atrophy in recurrent major depression. Proc. Natl. Acad. Sci. U S A 93, 3908-3913. doi: 10.1073/pnas.93.9.3908

Silva, J. M., Rodrigues, S., Sampaio-Marques, B., Gomes, P., Neves-Carvalho, A., Dioli, C., et al. (2019). Dysregulation of autophagy and stress granule-related proteins in stress-driven Tau pathology. Cell Death Differ. 26, 1411-1427. doi: 10.1038/s41418-018-0217-1

Sotiropoulos, I., Cerqueira, J. J., Catania, C., Takashima, A., Sousa, N., and Almeida, O. F. (2008). Stress and glucocorticoid footprints in the brain-the path from depression to Alzheimer's disease. Neurosci. Biobehav. Rev. 32, 1161-1173. doi: 10.1016/j.neubiorev.2008.05.007

Starkman, M. N., Gebarski, S. S., Berent, S., and Schteingart, D. E. (1992). Hippocampal formation volume, memory dysfunction and cortisol levels in patients with Cushing's syndrome. Biol. Psychiatry 32, 756-765. doi: 10.1016/0006-3223(92)90079-f

Starkman, M. N., Giordani, B., Berent, S., Schork, M. A., and Schteingart, D. E. (2001). Elevated cortisol levels in Cushing's disease are associated with cognitive decrements. Psychosom. Med. 63, 985-993. doi: 10.1097/00006842200111000-00018

Starkman, M. N., Giordani, B., Gebarski, S. S., and Schteingart, D. E. (2003). Improvement in learning associated with increase in hippocampal formation volume. Biol. Psychiatry 53, 233-238. doi: 10.1016/s0006-3223(02)01750-x

Tully, T., Bourtchouladze, R., Scott, R., and Tallman, J. (2003). Targeting the CREB pathway for memory enhancers. Nat. Rev. Drug Discov. 2, 267-277. doi: $10.1038 /$ nrd1061

Turnbull, A. V., and Rivier, C. L. (1999). Regulation of the hypothalamic-pituitaryadrenal axis by cytokines: actions and mechanisms of action. Physiol. Rev. 79, 1-71. doi: 10.1152/physrev.1999.79.1.1

Umegaki, H., Ikari, H., Nakahata, H., Endo, H., Suzuki, Y., Ogawa, O., et al. (2000). Plasma cortisol levels in elderly female subjects with Alzheimer's disease: a cross-sectional and longitudinal study. Brain Res. 881, 241-243. doi: 10.1016/s0006-8993(00)02847-X

Wang, Y., Li, M., Tang, J., Song, M., Xu, X., Xiong, J., et al. (2011). Glucocorticoids facilitate astrocytic amyloid- $\beta$ peptide deposition by increasing the expression of APP and BACE1 and decreasing the expression of amyloid- $\beta$-degrading proteases. Endocrinology 152, 2704-2715. doi: 10.1210/en.2011-0145

Wei, Q., Hebda-Bauer, E. K., Pletsch, A., Luo, J., Hoversten, M. T., Osetek, A. J., et al. (2007). Overexpressing the glucocorticoid receptor in forebrain causes an aging-like neuroendocrine phenotype and mild cognitive dysfunction. J. Neurosci. 27, 8836-8844. doi: 10.1523/JNEUROSCI.0910-07.2007

Wingenfeld, K., and Wolf, O. T. (2014). Stress, memory and the hippocampus. Front. Neurol. Neurosci. 34, 109-120. doi: 10.1159/000356423

Zalewska, K., Ong, L. K., Johnson, S. J., Nilsson, M., and Walker, F. R. (2017). Oral administration of corticosterone at stress-like levels drives microglial but not vascular disturbances post-stroke. Neuroscience 352, 30-38. doi: 10.1016/j. neuroscience.2017.03.005

Zhang, B., Xu, X., Niu, F., Mao, X., Dong, J., Yang, M., et al. (2020). Corticosterone replacement alleviates hippocampal neuronal apoptosis and spatial memory impairment induced by dexamethasone via promoting brain corticosteroid receptor rebalance after traumatic brain injury. J. Neurotrauma 37, 262-272. doi: $10.1089 /$ neu. 2019.6556

Conflict of Interest: The authors declare that the research was conducted in the absence of any commercial or financial relationships that could be construed as a potential conflict of interest.

Copyright (c) 2021 Zhang, Cao, Liang and Wang. This is an open-access article distributed under the terms of the Creative Commons Attribution License (CC BY). The use, distribution or reproduction in other forums is permitted, provided the original author(s) and the copyright owner(s) are credited and that the original publication in this journal is cited, in accordance with accepted academic practice. No use, distribution or reproduction is permitted which does not comply with these terms. 